\title{
Enantiomers of Single-Wall Carbon Nanotubes Show Distinct Coating Displacement Kinetics
}

\author{
Yu Zheng, ${ }^{\dagger}$ Sergei M. Bachilo, ${ }^{\dagger}$ and R. Bruce Weisman* ${ }^{*, \neq}$ \\ ${ }^{\dagger}$ Department of Chemistry and the Smalley-Curl Institute and ${ }^{\ddagger}$ Department of Materials Science \\ and NanoEngineering, Rice University, 6100 Main Street, Houston, Texas 77005, United States
}

\begin{abstract}
It is known that specific oligomers of single-stranded DNA (ssDNA) can show remarkable selectivity when coating different structural species of single-wall carbon nanotubes (SWCNTs). We report that (ATT) $)_{4}$ ssDNA coatings strongly distinguish between the two optical isomers of $(7,5)$ SWCNTs. This causes resolvable shifts in their fluorescence spectra and differences of two orders of magnitude in the room temperature rates of coating displacement, as monitored through changes in nanotube fluorescence wavelength and intensity on exposure to sodium deoxycholate. During coating displacement, the enantiomer with high affinity for the ssDNA oligomer is deduced to form an intermediate hybrid that is not observed for the low affinity enantiomer. These results reveal that enantiomeric differences in SWCNTs complexed with ssDNA are more diverse and dramatic than previously recognized.
\end{abstract}

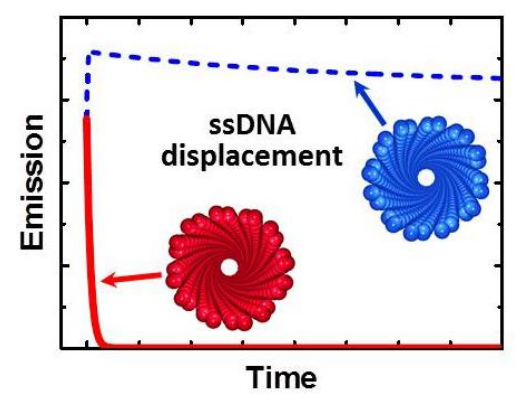


Single-wall carbon nanotubes (SWCNTs) are a family of tubular nanomaterials that resemble rolled-up sections of graphene sheets. A variety of possible SWCNT structures exist. Each of these is designated by a pair of integers, $(n, m)$, and has long-range crystalline order with a welldefined diameter and roll-up angle. ${ }^{1,2}$ Because the $\pi$-electron bands depend on SWCNT transverse structure, each $(n, m)$ species also has a distinct electronic character. Most are semiconducting and emit characteristic short-wave infrared fluorescence corresponding to their structure-specific band gaps. ${ }^{3,4}$ Nearly all $(n, m)$ species are also chiral because they comprise two non-superimposable mirror image forms corresponding to right- and left-handed helices (termed $\mathrm{P}$ and $\mathrm{M}) .{ }^{5-7}$ Although these enantiomers have identical intrinsic electronic properties, they may be physically separated through their differing interactions with chiral materials. ${ }^{8-10}$ Recently, Zheng and coworkers have efficiently sorted SWCNT enantiomers coated with single-stranded DNA (ssDNA) using aqueous two-phase partitioning. ${ }^{11}$ We find that ssDNA coatings can also greatly assist the separation of SWCNT enantiomers in nonlinear density-gradient ultracentrifugation. The major new finding reported here is that the structure-specific noncovalent interactions in a particular ssDNA-SWCNT hybrid show strong enough enantiomeric differences to cause resolvable spectral shifts in SWCNT fluorescence and dramatic kinetic differences in coating displacement.

According to the ssDNA-assisted sorting report of Zheng and co-workers and our recent discovery of selective fluorescence quenching by dissolved $\mathrm{O}_{2}$ in ssDNA-coated SWCNTs, $(7,5)$ SWCNTs show specific recognition by ssDNA oligos with the sequence (ATT) ${ }_{4 \cdot}{ }^{11-14}$ To further explore this unusual interaction, we suspended HiPco SWCNTs in aqueous (ATT) $)_{4}$ solution with the $\mathrm{pH}$ stabilized at 7.4 by buffering. Figure 1a shows the fluorescence spectrum of this sample. It contains a familiar superposition of emission features from a variety of semiconducting $(n, m)$ species whose peak wavelengths, spectral widths, and shapes differ slightly from those found in prior studies with more common surfactants. Using the approach developed previously, ${ }^{15}$ we 
analyzed the sample's emission spectrum to obtain the accurate simulation plotted as the red curve in Figure 1a. The underlying components deduced in this fitting showed one anomaly: a surprisingly large full-width at half-maximum of more than $30 \mathrm{~nm}$ for the $(7,5)$ feature, as compared to widths of 22.5 to $28.5 \mathrm{~nm}$ for other $(n, m)$ peaks in the spectrum. We found that an accurate spectral fit was also possible assuming two distinct $(7,5)$ emission features with plausible widths of 26 and $25 \mathrm{~nm}$ and peaks at 1044 and $1055 \mathrm{~nm}$.

Such a splitting of the $(7,5)$ emission might arise if the $(\text { ATT })_{4}$ ss-DNA coatings led to significantly different dielectric environments for the two enantiomers. In this view, the (ATT) 4 ssDNA oligos more effectively coat the high affinity $(7,5)$ enantiomer, denoted as $(7,5)_{\mathrm{HA}}$, than the lower affinity enantiomer, denoted as $(7,5)_{\text {LA. }}$ This effect should also lead to different buoyant densities of DNA- $(7,5)_{\mathrm{HA}}$ and DNA-(7,5)LA. To test this, we applied nonlinear densitygradient ultracentrifugation to the $(\mathrm{ATT})_{4}$-suspended sample and successfully extracted one $(7,5)$ band, which appeared as a distinct green layer in the upper part of the ultracentrifuge tube (see Figure S1). ${ }^{16}$ That component, termed DNA-(7,5) HA, was thus found to have a significantly lower buoyant density than the other species. Figure $1 \mathrm{~b}$ shows the fluorescence spectrum of the extracted DNA- $(7,5)_{\mathrm{HA}}$. The main feature is a peak at $1055 \mathrm{~nm}$ whose position and width closely match the longer wavelength $(7,5)$ emission component deduced by analyzing the spectrum of unsorted SWCNTs suspended in $(\mathrm{ATT})_{4}$ (Figure 1a). This agreement confirms that the unusual $(7,5)$ emission in that spectrum arises from two $(7,5)$ subspecies. We assign these to the nanotube enantiomers, with emission peaks of $1055 \mathrm{~nm}$ and $1044 \mathrm{~nm}$ (DNA-(7,5) $\mathrm{HA}$ and DNA-(7,5) $\mathrm{LA}$, respectively).

Further experiments revealed that the difference in coating environment that causes the $11 \mathrm{~nm}$ $\left(100 \mathrm{~cm}^{-1}\right)$ spectral splitting gives an even more dramatic difference in coating displacement kinetics. We measured time-sequenced fluorescence spectra after adding a solution of sodium deoxycholate (SDC), a strong nanotube surfactant, to the unsorted (ATT) $)_{4}$-suspended SWCNT 
mixture. Figure 2 plots the results, which are more complex than reported from prior kinetic spectroscopy studies of ssDNA displacement from SWCNTs. ${ }^{17-20}$ Within ca. $100 \mathrm{~s}$, most $(n, m)$ emission peaks had shifted to shorter wavelengths and intensified, an expected consequence when SDC displaces ssDNA on the nanotube surface. However, the spectral region near the $(7,5)$ emission feature (shown expanded in the figure inset) displayed much more complex changes over a range of time scales.

To investigate these changes, we analyzed kinetic spectra in the region of $(7,5)$ emission by careful deconvolution to find the underlying components and their magnitudes as a function of time. Figure 3 shows such deconvolutions at four time points during the first $100 \mathrm{~s}$ of coating displacement. As listed in Table 1, we found spectral components centered at 1035, 1044, and approximately $1054 \mathrm{~nm}$. Figure 4 plots the time dependence of these three emission spectra (note that the time scale of the top frame is 100 times shorter than the other two frames). The rapid decrease of the $1044 \mathrm{~nm}$ feature (top frame) is interpreted as the loss of the DNA-coated lower affinity enantiomer as its coating is displaced by SDC. The $1035 \mathrm{~nm}$ feature (bottom frame) appears within 3 seconds and grows with both rapid and slow components. We assign this feature to SDC-coated $(7,5)$ SWCNTs of both subspecies (although SDC is a chiral surfactant, it does not cause a resolvable shift between emission peaks of the enantiomers). We hypothesize that the rapid $1035 \mathrm{~nm}$ kinetic component corresponds to displacement of ssDNA from the lower affinity enantiomer. Emission near $1054 \mathrm{~nm}$, plotted in the center frame, also shows bimodal behavior. It quickly increases in intensity by ca. $20 \%$ during the first 15 seconds and then decreases slowly to an asymptotic level as the peak wavelength shifts slightly from 1055 to 1053 $\mathrm{nm}$. We assign this feature to the high affinity $(7,5)$ enantiomer, which is coated initially with $(\mathrm{ATT})_{4}$ ssDNA. In our model, the rapid increase in its emission intensity reflects the formation of a secondary coating of SDC, which may fill some of the gaps in the DNA surface coverage or adsorb as an additional layer. The subsequent intensity decrease near $1054 \mathrm{~nm}$ is caused by a 
blue shift of the emission peak to $1035 \mathrm{~nm}$ as SDC becomes the inner coating in a reversible displacement that leaves DNA in an outer layer (see step with $k_{3}$ in the scheme below).

These processes are summarized in the following proposed kinetic scheme:

$$
\begin{aligned}
& \mathrm{SDC}+\mathrm{DNA}^{-}(7,5)_{\mathrm{LA}} \stackrel{k_{1}}{\longrightarrow} \mathrm{SDC}-(7,5)_{\mathrm{LA}}+\mathrm{DNA} \\
& \mathrm{SDC}+\mathrm{DNA}(7,5)_{\mathrm{HA}} \stackrel{k_{2}}{\longrightarrow} \text { SDC/DNA-(7,5) } \mathrm{HA}_{k_{-3}} \stackrel{k_{3}}{\rightleftarrows} \text { DNA/SDC- }(7,5)_{\mathrm{HA}}
\end{aligned}
$$

Here, $k_{1}$ and $k_{2}$ are pseudo-first order rate constants that depend on the concentration of free SDC, and $k_{3}$ and $k_{-3}$ describe the reversible reorganization of mixed coatings of SDC and DNA on the high affinity $(7,5)$ nanotubes. Note that because our SWCNT samples likely contain a variety of specific surface coating structures for each species in the scheme above, the use of simple kinetic models with precisely defined rate constants will be less successful than for homogenous molecular systems. Nevertheless, the model shown above allows adequate kinetic fits to the data. These are shown as the computed solid curves in Figure 4. The deduced rate constant values are $0.067 \mathrm{~s}^{-1}$ for $k_{1}, 0.30 \mathrm{~s}^{-1}$ for $k_{2}, 5.8 \times 10^{-4} \mathrm{~s}^{-1}$ for $k_{3}$, and $4.6 \times 10^{-4} \mathrm{~s}^{-1}$ for $k_{-3}$. Our simulations also use a value of 1.3 (determined from the peak in Figure $4 \mathrm{~b}$ ) for the emissive quantum yield ratio of SDC/DNA- $(7,5)_{\mathrm{HA}}$ compared to DNA- $(7,5)_{\mathrm{HA}}$, and a value of 0.38 for the initial concentration ratio $\frac{\left[\operatorname{DNA}-(7,5)_{\mathrm{LA}}\right]_{0}}{\left[\operatorname{DNA}-(7,5)_{\mathrm{HA}}\right]_{0}}$. The spectral deconvolution shown in Figure 1a indicates that the product of initial concentration and emissive quantum yield is greater for the high affinity than for the low affinity species in our $\mathrm{ATT}_{4}$-dispersed sample, although we do not know the separate roles of these factors. In our ambient temperature measurements, the ratio of time scales for displacement, corresponding to $k_{1} / k_{3}$, is approximately 115 . Assuming similar kinetic prefactors, this suggests an activation energy difference of ca. $12 \mathrm{~kJ} / \mathrm{mol}\left(5 k_{\mathrm{B}} \mathrm{T}\right)$ between enantiomers. This result may aid computational efforts to clarify the energetic factors controlling SWCNT enantiomer recognition by ssDNA. ${ }^{18,21,22}$ 
The pseudo-first order rate constants $k_{1}$ and $k_{2}$ given above were deduced for an initial SDC concentration of $4.8 \times 10^{-4} \mathrm{M}$. Surprisingly, we observed a highly nonlinear dependence of these rate constants on SDC concentration, with a kinetic partial order estimated as approximately 5 (see Supporting Information). We suggest that this reflects the dominant role of small molecular clusters of SDC, rather than individual molecules, in the coating displacement mechanism. Although it is not included in the kinetic scheme, higher SDC concentrations (such as used for the run of Figure 2) or longer incubations lead to the irreversible conversion of DNA- $(7,5)_{\mathrm{HA}}$ into SDC- $(7,5)_{\text {HA. }}$

We have previously reported that dissolved $\mathrm{O}_{2}$ can partially quench the fluorescence of SWCNTs in aqueous ssDNA dispersions, and that the extent of this quenching reveals selective affinities between specific ssDNA oligos and specific $(n, m)$ species. ${ }^{14}$ Consistent with the kinetic findings described above, we observe large differences in dissolved oxygen fluorescence quenching of DNA- $(7,5)_{\mathrm{LA}}$ compared to DNA- $(7,5)_{\mathrm{HA}}$. The quenching is much stronger for the DNA- $(7,5)_{\mathrm{LA}}$ enantiomer, as expected for less complete coverage of the nanotube surface and greater exposure to dissolved species. Details of enantiomer-specific $\mathrm{O}_{2}$ quenching will be reported separately.

In summary, we have used fluorescence spectroscopy to uncover strongly enantiomerspecific coating displacement kinetics for $(7,5)$ SWCNTs suspended by ssDNA oligos with the structure $(\mathrm{ATT})_{4}$. The enantiomeric selectivity of this ssDNA coating creates two subpopulations with distinct emission wavelengths and buoyant densities, in addition to dramatically different rates and mechanisms of displacement by added SDC. While the lower affinity enantiomer is displaced in seconds by ca. $5 \times 10^{-4} \mathrm{M} \mathrm{SDC}$, the higher affinity enantiomer is deduced to quickly acquire a secondary coating of SDC that dislodges the ssDNA layer on a time scale approximately 100 times longer than displacement from the lower affinity form. This study 
shows that the wide range of physicochemical properties displayed by single-wall carbon nanotubes is significantly expanded by distinguishing their left- and right-handed enantiomers.

\section{ASSOCIATED CONTENT}

\section{Supporting Information}

Details of materials and sample preparation; nonlinear DGU sorting; photoluminescence measurements; and effect of SDC concentration on displacement kinetics. The Supporting Information is available free of charge on the ACS Publications website.

\section{AUTHOR INFORMATION}

\section{Corresponding Author}

weisman@ rice.edu. Tel: 713-3483709. Fax: 713-348-5155.

\section{Notes}

The authors declare the following competing financial interest: R.B.W. has a financial interest in Applied NanoFluorescence, LLC, which manufactures one of the instruments used in this study.

\section{ACKNOWLEDGEMENTS}

This research was supported by grants from the National Science Foundation (CHE-1409698) and the Welch Foundation (C-0807). We thank S. R. Sanchez for helpful discussions.

\section{REFERENCES}

(1) Saito, R.; Dresselhaus, G.; Dresselhaus, M. S., Physical Properties of Carbon Nanotubes; Imperial College Press: 1998.

(2) Reich, S.; Thomsen, C.; Maultzsch, J., Carbon Nanotubes: Basic Concepts and Physical Properties; Wiley-VCH: Weinheim, 2004.

(3) Bachilo, S. M.; Strano, M. S.; Kittrell, C.; Hauge, R. H.; Smalley, R. E.; Weisman, R. B. Structure-Assigned Optical Spectra of Single-Walled Carbon Nanotubes. Science 2002, 298, 2361-2366.

(4) Weisman, R. B.; Bachilo, S. M. Dependence of Optical Transition Energies on Structure for Single-Walled Carbon Nanotubes in Aqueous Suspension: An Empirical Kataura Plot. Nano Lett. 2003, 3, 1235-1238. 
(5) Ivchenko, E. L.; Spivak, B. Chirality Effects in Carbon Nanotubes. Phys. Rev. B 2002, 66, 1554041-1554049.

(6) Samsonidze, G. G.; Gruneis, A.; Saito, R.; Jorio, A.; Souza Filho, A. G.; Dresselhaus, G.;

Dresselhaus, M. S. Interband Optical Transitions in Left- and Right-Handed Single-Wall Carbon Nanotubes. Phys. Rev. B 2004, 69, 205402.

(7) Sanchez-Castillo, A.; Noguez, C. Understanding Optical Activity in Single-Walled Carbon Nanotubes from First-Principles Studies. J. Phys. Chem. C 2010, 114, 9640-9644.

(8) Ghosh, S.; Bachilo, S. M.; Weisman, R. B. Structure Dependent Optical Activity of Single-Walled Carbon Nanotube Enantiomers. Fullerenes, Nanotubes, Carbon Nanostruct. 2014, 22, 269-279.

(9) Green, A. A.; Duch, M. C.; Hersam, M. C. Isolation of Single-Walled Carbon Nanotube Enantiomers by Density Differentiation. Nano Res. 2009, 2, 69-77.

(10) Peng, X.; Komatsu, N.; Kimura, T.; Osuka, A. Improved Optical Enrichment of SWNTs through Extraction with Chiral Nanotweezers of 2,6-Pyridylene-Bridged Diporphyrins. J. Am. Chem. Soc. 2007, 129, 15947-15953.

(11) Ao, G.; Streit, J. K.; Fagan, J. A.; Zheng, M. Differentiating Left- and Right-Handed Carbon Nanotubes by DNA. J. Am. Chem. Soc. 2016, 138, 16677-16685.

(12) Tu, X.; Manohar, S.; Jagota, A.; Zheng, M. DNA Sequence Motifs for Structure-Specific Recognition and Separation of Carbon Nanotubes. Nature 2009, 460, 250-253.

(13) Ao, G.; Khripin, C. Y.; Zheng, M. DNA-Controlled Partition of Carbon Nanotubes in Polymer Aqueous Two-Phase Systems. J. Am. Chem. Soc. 2014, 136, 10383-10392.

(14) Zheng, Y.; Bachilo, S. M.; Weisman, R. B. Quenching of Single-Walled Carbon Nanotube Fluorescence by Dissolved Oxygen Reveals Selective Single-Stranded DNA Affinities. J. Phys. Chem. Lett. 2017, 8, 1952-1955.

(15) Rocha, J. D.; Bachilo, S. M.; Ghosh, S.; Arepalli, S.; Weisman, R. B. Efficient Spectrofluorimetric Analysis of Single-Walled Carbon Nanotube Samples. Anal. Chem. 2011, 83, 7431-7437.

(16) Ghosh, S.; Bachilo, S. M.; Weisman, R. B. Advanced Sorting of Single-Walled Carbon Nanotubes by Nonlinear Density-Gradient Ultracentrifugation. Nat. Nanotechnol. 2010, 5, 443-450.

(17) Roxbury, D.; Tu, X.; Zheng, M.; Jagota, A. Recognition Ability of DNA for Carbon Nanotubes Correlates with Their Binding Affinity. Langmuir 2011, 27, 8282-8293. 
(18) Shankar, A.; Mittal, J.; Jagota, A. Binding between DNA and Carbon Nanotubes Strongly Depends Upon Sequence and Chirality. Langmuir 2014, 30, 3176-3183.

(19) Jena, P. V.; Safaee, M. M.; Heller, D. A.; Roxbury, D. DNA-Carbon Nanotube Complexation Affinity and Photoluminescence Modulation Are Independent. ACS Appl. Mater. Interfaces 2017, 9, 21397-21405.

(20) Kato, Y.; Inoue, A.; Niidome, Y.; Nakashima, N. Thermodynamics on Soluble Carbon Nanotubes: How Do DNA Molecules Replace Surfactants on Carbon Nanotubes? Sci. Rep. 2012, 2, 733.

(21) Shankar, A.; Zheng, M.; Jagota, A. Energetic Basis of Single-Wall Carbon Nanotube Enantiomer Recognition by Single-Stranded DNA. J. Phys. Chem. C 2017, 121, 1747917487.

(22) Roxbury, D.; Mittal, J.; Jagota, A. Molecular-Basis of Single-Walled Carbon Nanotube Recognition by Single-Stranded DNA. Nano Lett. 2012, 12, 1464-1469. 
Table 1. $(7,5)$ SWCNT Species Involved in the Coating Displacement Study

\begin{tabular}{|c|c|c|c|c|c|}
\hline species & symbol & $\begin{array}{l}\text { emission } \\
\text { peak }(\mathrm{nm})\end{array}$ & $\begin{array}{l}\text { relative } \\
\text { quantum } \\
\text { yield }\end{array}$ & kinetics & notes \\
\hline $\begin{array}{l}(7,5) \text { enantiomer } 1 \\
\text { in }(\mathrm{ATT})_{4} \text { SSDNA }\end{array}$ & DNA- $(7,5)_{\llcorner A}$ & 1044 & low & fast decay & low affinity hybrid \\
\hline $\begin{array}{l}(7,5) \text { enantiomer } 2 \\
\text { in }(\mathrm{ATT})_{4} \text { SsDNA }\end{array}$ & DNA- $(7,5)_{\mathrm{HA}}$ & 1055 & low & complex & high affinity hybrid \\
\hline $\begin{array}{l}(7,5) \text { enantiomer } 1 \\
\text { in SDC }\end{array}$ & SDC- $(7,5)_{\llcorner A}$ & 1035 & high & fast rise & $\begin{array}{l}\text { enantiomer } 2 \text { in sodium } \\
\text { deoxycholate }\end{array}$ \\
\hline $\begin{array}{l}(7,5) \text { enantiomer } 2 \\
\text { in SDC and }(A T T)_{4}\end{array}$ & DNA/SDC- $(7,5)_{\mathrm{HA}}$ & 1035 & high & slow rise & $\begin{array}{l}\text { enantiomer } 1 \text { with primary } \\
\text { coating of sodium } \\
\text { deoxycholate, } \\
\text { secondary of DNA }\end{array}$ \\
\hline $\begin{array}{l}(7,5) \text { enantiomer } 2 \\
\text { in }(\mathrm{ATT})_{4} \text { and SDC }\end{array}$ & SDC/DNA- $(7,5)_{\mathrm{HA}}$ & 1053 & medium & fast rise & $\begin{array}{l}\text { enantiomer } 1 \text { with primary } \\
\text { coating of DNA, } \\
\text { secondary of sodium } \\
\text { deoxycholate }\end{array}$ \\
\hline
\end{tabular}




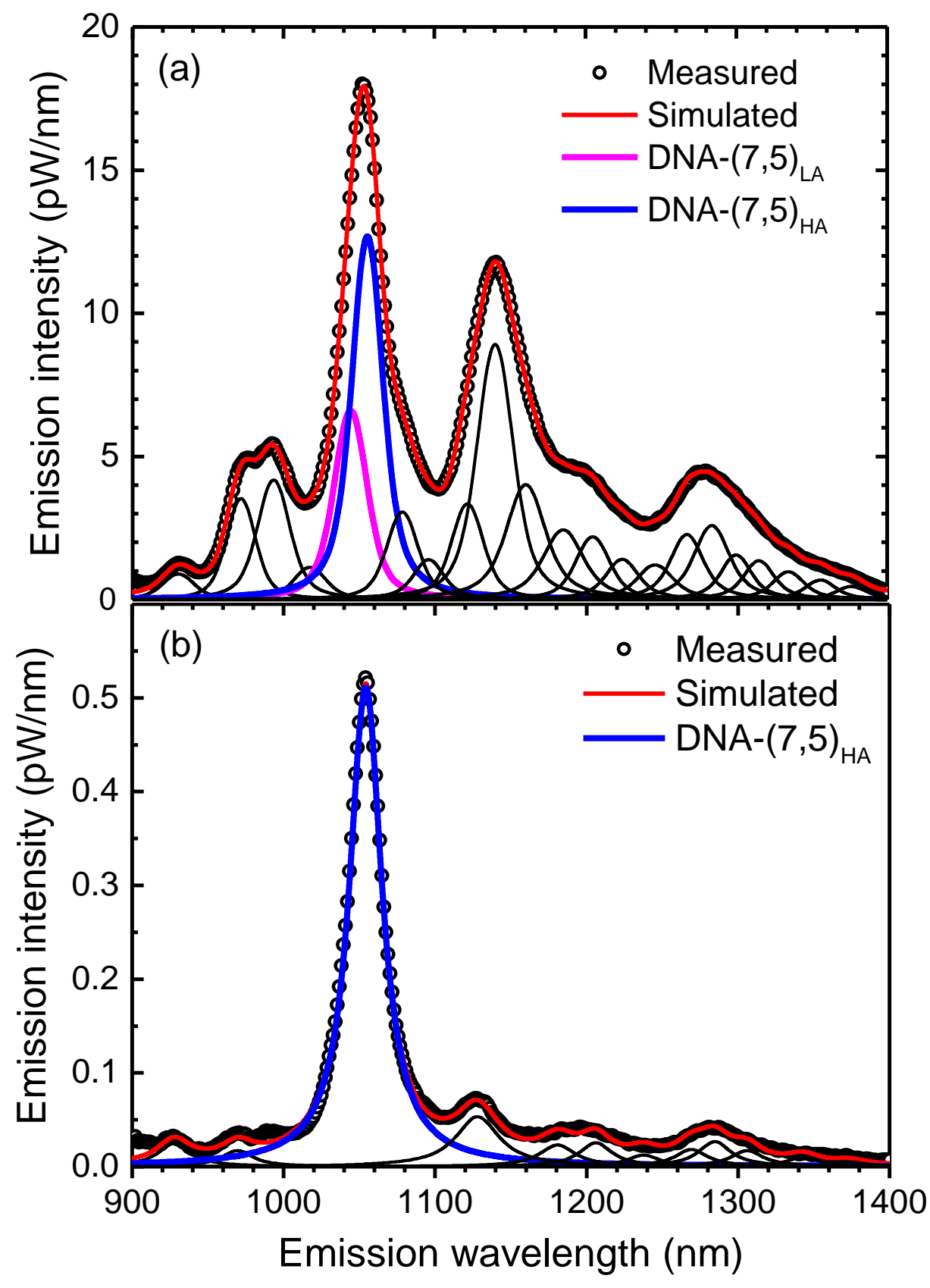

Figure 1. (a) Measured (symbols) and simulated (solid red curve) fluorescence spectrum of an unsorted bulk SWCNT sample suspended in (ATT) $)_{4}$ ssDNA solution (642 nm excitation). Thin black curves show the deduced emission components from different $(n, m)$ species. Components between 900 and $1200 \mathrm{~nm}$ represent specific known $(n, m)$ species, but those between 1200 and $1400 \mathrm{~nm}$ do not. Deduced emission spectra assigned to the two $(7,5)$ enantiomers are drawn in pink and blue. (b) Fluorescence spectrum of a $(7,5)$-enriched SWCNT sample suspended in $(\mathrm{ATT})_{4}$ solution. The spectrum is dominated by the component shown as a blue trace (DNA$\left.(7,5)_{\mathrm{HA}}\right)$. 


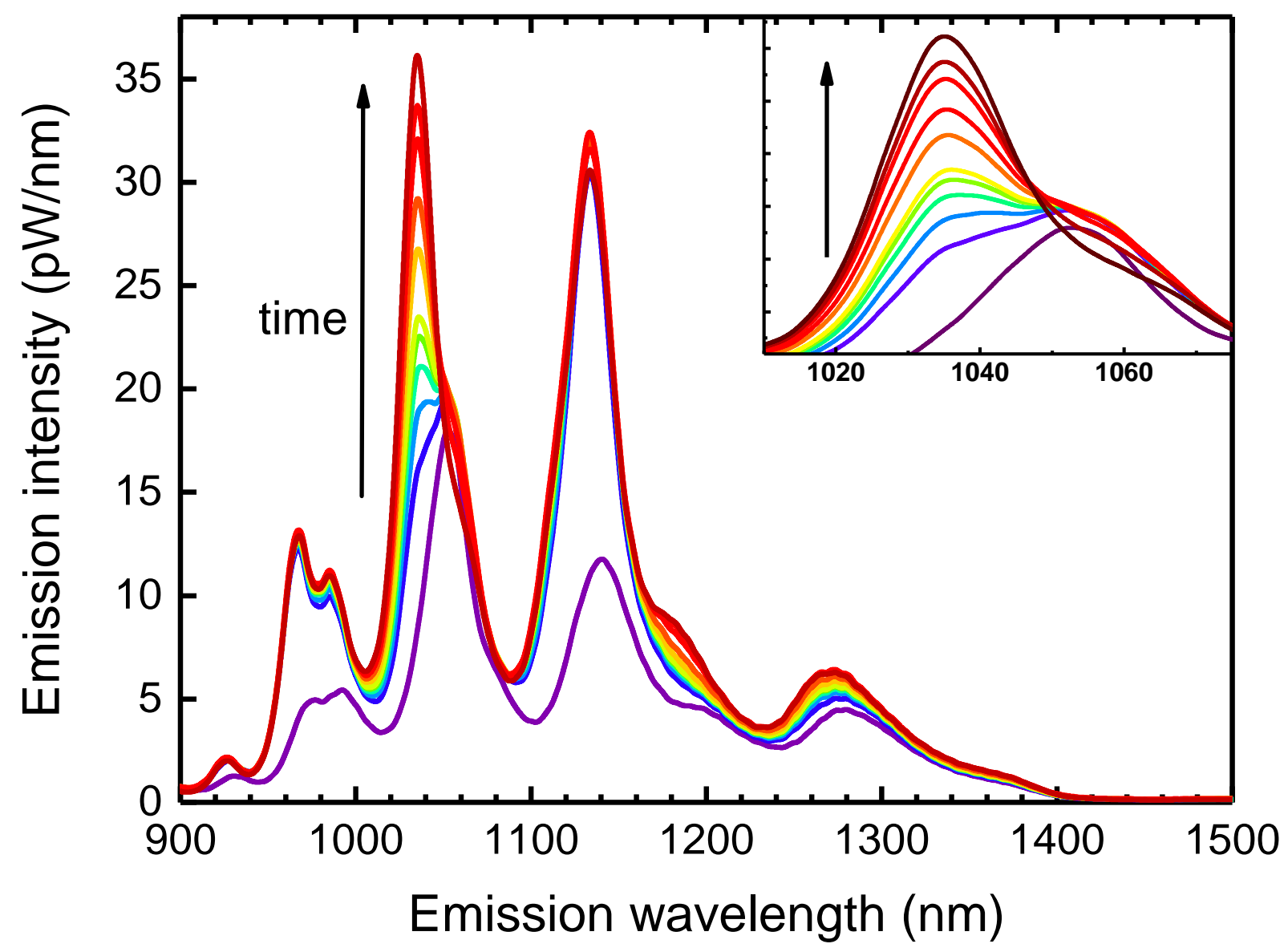

Figure 2. Time-resolved fluorescence spectra for SWCNTs dispersed in $(\mathrm{ATT})_{4}$ during displacement by SDC. The traces were measured at 0, 1, 2, 3, 4, 5, 10, 30, 150, 390, and 800 minutes after exposure to $6.3 \times 10^{-4} \mathrm{M}$ SDC. The inset shows spectral changes in the region of $(7,5)$ emission. 

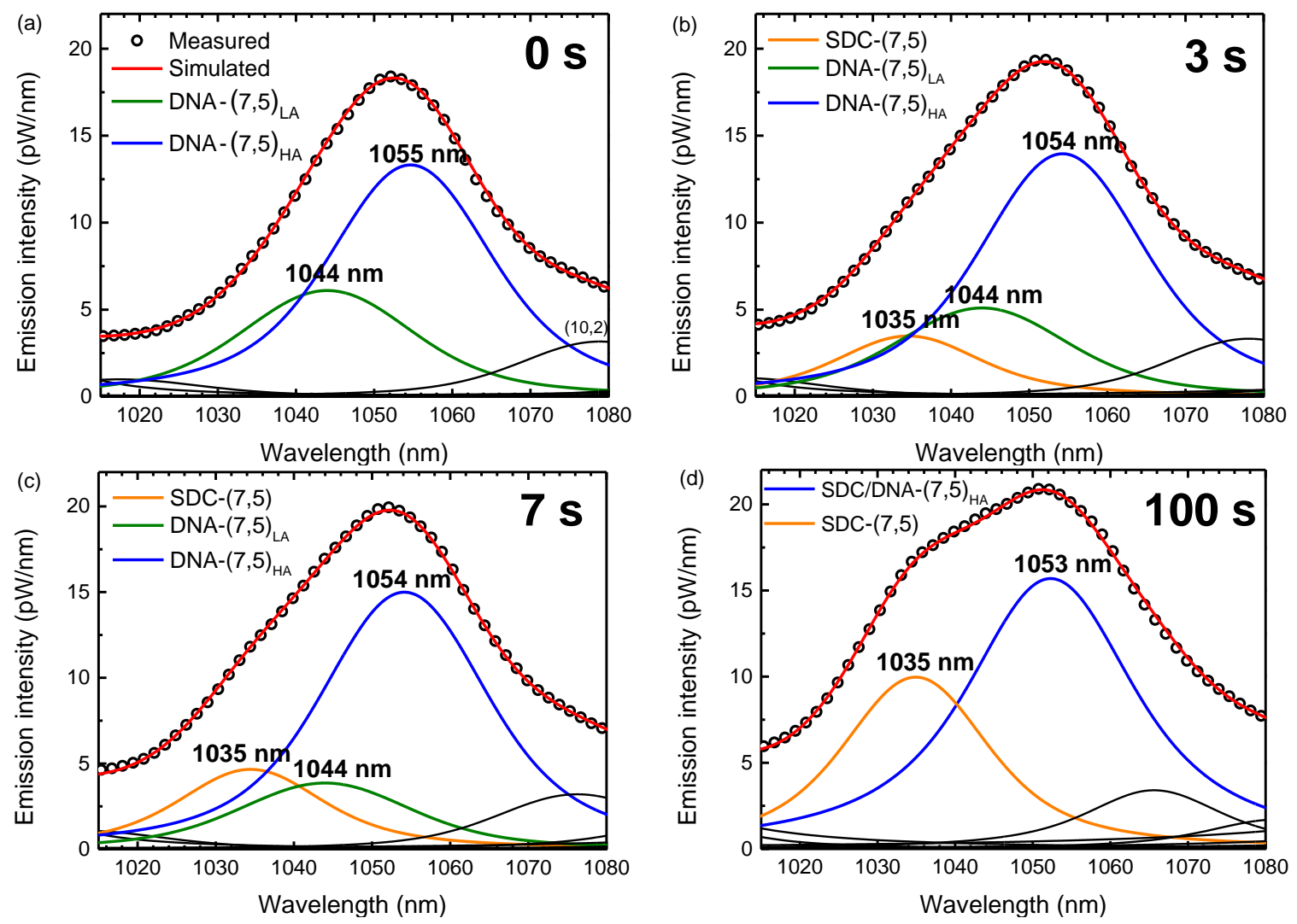

Figure 3. Measured (symbols) and simulated (solid red curves) fluorescence spectra of a bulk SWCNT sample in the range of $(7,5)$ emission. The green curve represents the emission from low affinity DNA- $(7,5)_{\mathrm{LA}}$; the blue curve represents the emission from high affinity DNA$(7,5)_{\mathrm{HA}}$; the orange curve represents the emission from SDC-(7,5). (a) Emission from SWCNTs suspended in (ATT) 4 solution just before SDC addition, (b) at $3 \mathrm{~s}$ after addition, (c) at $7 \mathrm{~s}$, (d) at $100 \mathrm{~s}$. 

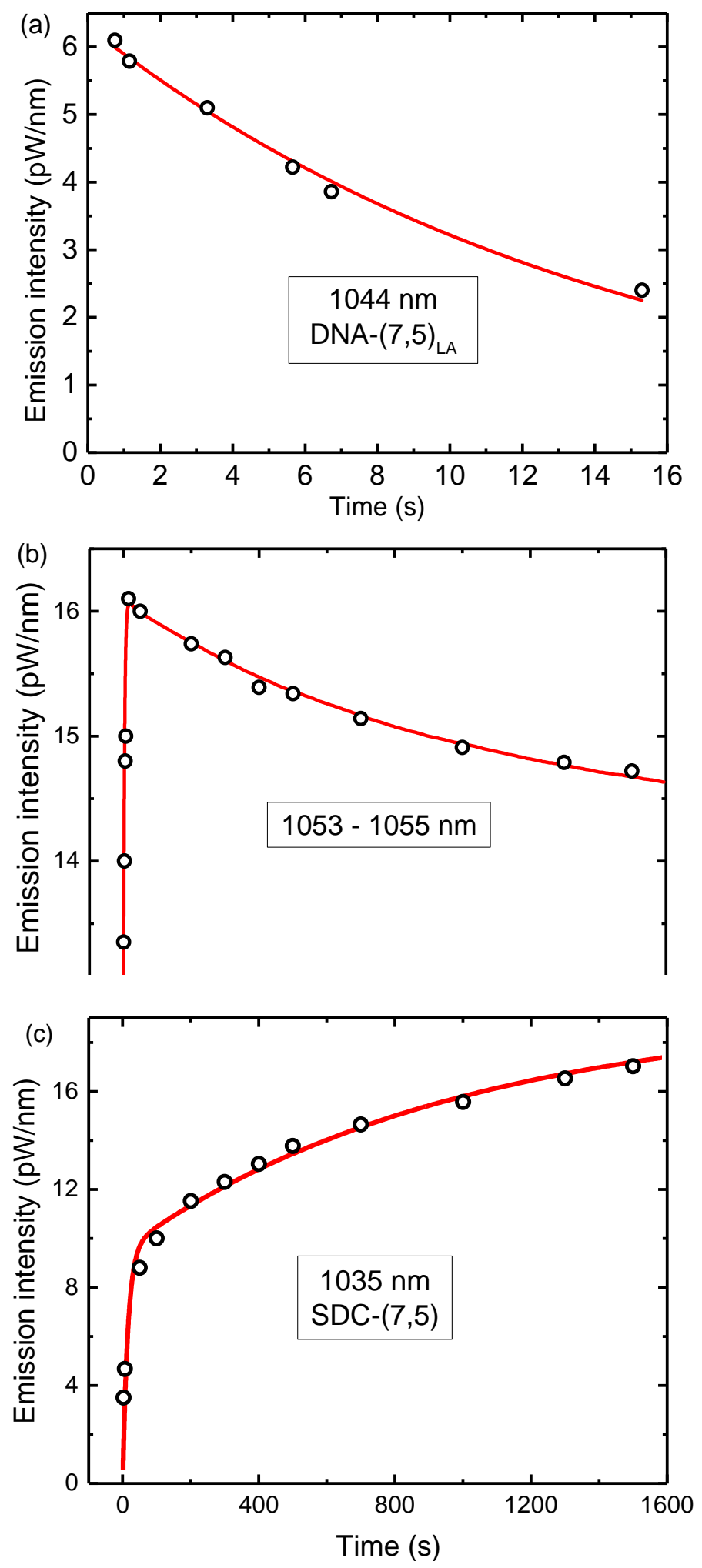

Figure 4. Deduced species-resolved fluorescence kinetics during displacement of ssDNA coating by SDC. (a) species with emission peak at $1044 \mathrm{~nm}$, assigned as DNA-(7,5) $\mathrm{LA}$; (b) species with emission peak near $1054 \mathrm{~nm}$; (c) species with emission peak at $1035 \mathrm{~nm}$, assigned as SDC-(7,5). Note that the time scale in (a) is 100 times shorter than in (b) and (c). Symbols are experimental points and solid curves show kinetic simulations using parameters given in the text. 